\title{
Evaluation of hyperbaric oxygen therapy for spinal cord injury in rats with different treatment course using diffusion tensor imaging
}

\author{
Fang Liu ${ }^{1} \cdot$ Libin Yang $^{2} \cdot$ Jianyi Liu ${ }^{2} \cdot$ Yijing Zhao $^{2} \cdot$ Zebin Xiao $^{2} \cdot$ Yingyan Zheng $^{2} \cdot$ Zhen Xing $^{2} \cdot$ Yuyang Zhang $^{2}$. \\ Dairong $\mathrm{CaO}^{2}$
}

Received: 25 August 2018 / Revised: 26 November 2018 / Accepted: 20 December 2018 / Published online: 14 January 2019

(C) International Spinal Cord Society 2019

\begin{abstract}
Study design Animal study.

Objectives To evaluate the efficacy of hyperbaric oxygen (HBO) therapy for spinal cord injury (SCI) in rats with different treatment course using diffusion tensor imaging (DTI).

Setting Hospital in Fuzhou, China.

Methods Fifty adult Sprague-Dawley rats were grouped as: (A) sham-operated group $(n=10)$; (B) SCI without HBO therapy group $(n=10)$; (C) SCI with $\mathrm{HBO}$ therapy for 2 weeks $\left(\mathrm{SCI}+\mathrm{HBO}_{2 \mathrm{~W}}\right)$ group $(n=10)$; (D) SCI with HBO therapy for 4 weeks $\left(\mathrm{SCI}+\mathrm{HBO}_{4 \mathrm{~W}}\right)$ group $(n=10)$; (E) $\mathrm{SCI}$ with $\mathrm{HBO}$ therapy for 6 weeks $\left(\mathrm{SCI}+\mathrm{HBO}_{6 \mathrm{~W}}\right)$ group $(n=10)$. Basso Beattie Bresnahan (BBB) scores and diffusion tensor imaging parameters including fractional anisotropy (FA), mean diffusivity (MD), radial diffusion (RD), and axial diffusion (AD) values in the injury epicenter, as well as $2 \mathrm{~mm}$ rostral and caudal to the injury epicenter were collected and analyzed 6 weeks post-injury.

Results Higher $\mathrm{BBB}$ score and FA values were found in the $\mathrm{SCI}+\mathrm{HBO}_{4 \mathrm{~W}}$ group than in the $\mathrm{SCI}$ and $\mathrm{SCI}+\mathrm{HBO}_{2 \mathrm{~W}}$ groups (all $P<0.05$ ), whereas no significant differences of these metrics were observed between the $\mathrm{SCI}+\mathrm{HBO}_{4 \mathrm{~W}}$ and $\mathrm{SCI}+\mathrm{HBO} 6 \mathrm{~W}$ groups. $\mathrm{MD}$ and $\mathrm{RD}$ values of the $\mathrm{SCI}+\mathrm{HBO}_{4 \mathrm{~W}}$ group were significantly lower than those of the $\mathrm{SCI}$ group (all $P<0.01$ ). FA values were positively correlated with $\mathrm{BBB}$ scores. $\mathrm{MD}$ and $\mathrm{RD}$ values were negatively correlated with $\mathrm{BBB}$ scores.

Conclusion DTI parameters, especially FA, could non-invasively and quantifiably evaluate the efficacy of HBO treatment for rats with SCI and 4 weeks may be the more appropriate treatment course.
\end{abstract}

\section{Introduction}

Spinal cord injury (SCI) is a common and severe injury in the central nervous system, which affects people in physical, psychological, and socioeconomical aspects at different degrees [1]. Notably, the pathophysiological changes of

These authors contributed equally: Fang Liu, Libin Yang

Fang Liu

913542386@qq.com

$\triangle$ Dairong Cao

dairongcao@163.com

1 Department of Hyperbaric Oxygen, The First Affiliated Hospital of Fujian Medical University, Fuzhou, Fujian, China

2 Department of Radiology, The First Affiliated Hospital of Fujian Medical University, Fuzhou, Fujian, China spinal cord after SCI usually involve primary and secondary damages [2]. Primary injury is often considered to be irreversible, but targeting some mechanisms of secondary injury has been proven to be helpful for improving the individuals' neurological outcomes [2]. Hyperbaric oxygen (HBO) therapy, in which $100 \%$ oxygen is administered at a pressure between one and three times that of atmospheric pressure, is a promising treatment which can effectively ameliorate secondary damage in spinal cord injury. To date, there are a number of studies concerning the mechanisms of HBO therapy for rats after SCI, but limited research focusing on the treatment course of HBO therapy for persons with SCI has been reported [3-7]. More importantly, the protocols of HBO therapy for patients with SCI is varying in different hospitals or medical institutions, leaving a great challenge to determine whether the prolonged periods for HBO therapy could have a beneficial effect on further improving functional outcome in individuals with SCI. Therefore, the standardization of HBO therapy is 
hindered, since no consensuses or guidelines on the treatment course of HBO therapy have been proposed. With this regard, we performed the present study attempting to seek for the best appropriate treatment course for rats with SCI by comparing the functional recovery in rats with different treatment course.

At present, the evaluation of functional recovery after HBO therapy for rats with SCI mainly relies on the histopathological analysis of excised spinal cord tissue. However, as an invasive technique, histopathological examination holds a limited capacity for the spatial and temporal evolution of SCI and cannot be conducted in humans. Therefore, there is a need to seek a non-invasive technique that can monitor the progression of injury and evaluate the efficacy of HBO treatment in a single individual, and can be translated to humans. Conventional MRI is a useful imaging modality for the routine examination of human SCI, as it can reflect pathological status such as hemorrhage, edema, and cavitation [8]. However, the morphological changes and the signal variations on the conventional MRI images after SCI are not reliable or accurate enough to assess the functional integrity of the injured spinal cord. Diffusion tensor imaging (DTI) is widely used as an efficient imaging technique to noninvasively quantify the diffusion of water molecules in tissues. DTI and the derived quantitative parameters including fractional anisotropy (FA), mean diffusivity (MD), radial diffusion (RD), and axial diffusion (AD) have been reported to be more sensitive than conventional MRI in detecting pathophysiology processes and predicting the severity of the injury after SCI [9-12]. FA refers to the anisotropy of diffusion, which is associated with the existence of axonal architecture, myelin sheaths, and cell membranes, and MD reflects the mean magnitude of diffusion. In a recent study reported by Sun et al. [13], FA values decreased but MD values increased significantly in the spinal cord of rat with SCI, whereas these two parameters were improved in rats with $\mathrm{HBO}$ treatment, revealing that these two parameters could serve as promising biomarkers to indicate the damage of the fiber integrity after SCI and to evaluate the efficacy of HBO treatment in SCI. Moreover, previous studies demonstrated that $\mathrm{AD}$ and $\mathrm{RD}$ reflected axonal damage [14] and demyelination [15], respectively, which could provide more information about the histopathological process of the spinal cord injury. In this regard, we hypothesis that DTI is a preferred method to comprehensively and non-invasively evaluate the function recovery in the rehabilitation process for rats after SCI.

Therefore, our study aimed to identify the most appropriate treatment course of HBO therapy for rats after SCI by measuring the changes of DTI parameters in rats with different treatment protocols.

\section{Methods}

\section{Animals}

A total of 50 healthy adult male Sprague-Dawley rats, aged 6-8 weeks and weighing $180-220 \mathrm{~g}$, were provided by the Experimental Animal Center of Fujian Medical University (Fujian, China). The rats had free access to food and water and were housed within a constant temperature and humidity environment in a normal $12 \mathrm{~h}$ light/dark cycles.

\section{Experimental groups}

Forty rats were randomly divided into four groups: (A) SCI without HBO therapy group (SCI, $n=10$ ); (B) SCI with $\mathrm{HBO}$ therapy for 2 weeks group $\left(\mathrm{SCI}+\mathrm{HBO}_{2 \mathrm{~W}}, n=10\right)$; (C) SCI with $\mathrm{HBO}$ therapy for 4 weeks group (SCI $+\mathrm{HBO}_{4 \mathrm{w}}, n=10$ ); (D) SCI with HBO therapy for 6 weeks group $\left(\mathrm{SCI}+\mathrm{HBO}_{6 \mathrm{~W}}, n=10\right)$. In addition, the shamoperated group ( $\mathrm{SH}, n=10)$ was added in the later stage.

\section{$\mathrm{SCl}$ model}

The experiment was approved by the Experimental Animal Ethics Committee of Fujian Medical University (No. 20170109). The SCI rat model was established according to the method previously described by Basso et al. [16]. All surgical procedures were performed under aseptic conditions. The rat was anesthetized with intraperitoneal injection of $2 \%$ pentobarbital sodium at a dose of $50 \mathrm{mg} / \mathrm{kg}$ and was placed in prone position. Laminectomy was performed in T9-10 vertebra to expose the spinal cord. A moderate SCI was created at T10 segment with the New York University (NYU) impactor by dropping a $10 \mathrm{~g}$ rod from a distance of $25 \mathrm{~mm}$. The characteristics of a successful model included: a wagging tail reflex in the rats, retraction of the lower limbs and flaccid paralysis of the lower extremities. Then, the incision was closed in layers and gentamicin was administered intramuscularly for prevention of postoperative infection. The bladder was compressed manually by abdominal pressure twice a day until the restoration of micturition reflex. There was one rat lost in the modeling process. The rats in the $\mathrm{SH}$ group received laminectomy only.

\section{HBO therapy}

Rats in the SCI $+\mathrm{HBO}$ groups were received $60 \mathrm{~min}$ of HBO therapy $\left(\mathrm{PO}_{2}=95-100 \%\right.$ at 2.0 ATA $)$ in an animal hyperbaric oxygen chamber (DC0325J-X, Yantai Moon Group Co., Ltd., Shandong, China) for $6 \mathrm{~h}$ after injury. The therapy was conducted twice per day at $8 \mathrm{~h}$ intervals for the first 3 days and then daily for the consecutive days. The chamber pressure was gradually increased to 2.0 ATA for 
10 min and depressurized to normobaric air $\left(\mathrm{PO}_{2}=21 \%\right.$ at 1.0 ATA) for $10 \mathrm{~min}$. The chamber temperature and humidity were maintained at $22^{\circ} \mathrm{C}-25^{\circ} \mathrm{C}$ and $62-68 \%$, respectively. Rats in the $\mathrm{SH}$ and SCI groups were exposed to normobaric air condition.

\section{Locomotor function assessment}

Three blinded individuals participated in the hindlimb locomotion testing for each rat at 6 weeks after SCI. Each animal was tested in an open field with the Basso, Beattie, Bresnahan (BBB) scale for $4 \mathrm{~min}$. The BBB scoring varied from 0 (paralysis) to 21 (normal gaits) with 22 categories. The output score of each rat was the average of locomotion scores of bilateral hind limbs.

\section{MRI and DTI acquisition}

The conventional MRI and DTI examinations were conducted in vivo for 6 weeks post-injury. All MRI examinations were performed on a 3.0 T MRI scanner (Magnetom Skyra; Siemens, Erlangen, Germany) by using a specialized animal coil (CG-MUC18-H300-AS, Shanghai chenguang medical technology Co., Ltd., China). Conventional MRI scan, including T1-weighted and T2-weighted images (T1WI and T2WI) was completed with the spin echo sequence. The axial T2WI was acquired with $\mathrm{TR} / \mathrm{TE}=2500 / 78 \mathrm{~ms}$, field of view $(\mathrm{FOV})=80 \mathrm{~mm} \times 60 \mathrm{~mm}$ and slice thickness $=$ $2.0 \mathrm{~mm}$. The sagittal T2WI and T1WI was obtained with TR/ $\mathrm{TE}=2070 / 79 \mathrm{~ms}$ and $\mathrm{TR} / \mathrm{TE}=1040 / 12 \mathrm{~ms}$ respectively, but FOV at $55 \mathrm{~mm} \times 55 \mathrm{~mm}$, slice thickness of $1.2 \mathrm{~mm}$ were uniform in the two sequences. Readout segmentation of long variable echo-trains (RESOLVE) sequence were used to perform DTI scan which had the same centering as the anatomical images and the relevant parameters were: $\mathrm{TR}=3130 \mathrm{~ms}, \mathrm{TE}=65 / 93 \mathrm{~ms}, \mathrm{FOV}=162 \mathrm{~mm} \times 81 \mathrm{~mm}$, matrix $=168 \times 168$, slice thickness $=2.0 \mathrm{~mm}, 20$ diffusion gradient directions with two $b$ values $\left(b=0\right.$ and $\left.800 \mathrm{~s} / \mathrm{mm}^{2}\right)$. Lesion site-centric FOV was performed with aforementioned sequences.

\section{DTI data processing}

After image acquisition, the data were transferred to an independent workstation (Siemens syngo MR VB10B) and the processing and analysis of data were performed by the inbuilt software (MR Neuro 3D). The diffusion tensor images were co-registered to the axial T2WI and then two experienced radiologists who were blinded to the study independently drew the regions of interest (ROIs) of the whole cord in the epicenter, rostral and caudal $2 \mathrm{~mm}$ slices. Care was taken to avoid partial contamination from the surrounding cerebrospinal fluid. Automatic calculation of
DTI parameters including FA, MD, RD, and AD values within the ROIs were carried out.

\section{Statistical analysis}

All statistical analyses were performed using SPSS version 23.0 (SPSS Inc., IL, USA). Data were expressed as mean \pm standard deviation (SD). Independent-samples $t$-test was used to evaluate statistical significance between the $\mathrm{SH}$ and SCI groups. One-way analysis of variance was used for multiple comparison of SCI and SCI $+\mathrm{HBO}$ groups at the injury epicenter, rostral and caudal levels respectively and Tukey honest significant difference test and Games-Howell test were used to evaluate significant differences between groups. Moreover, Bonferroni correction was performed for multiple comparisons. Spearman correlation analysis was applied to analyze the correlation between BBB scores and DTI parameters, including FA, MD, AD, and RD. $P$ value $<0.05$ was considered as statistically significant.

\section{Results}

\section{BBB score}

The BBB scores of the $\mathrm{SCI}+\mathrm{HBO}$ groups, including SCI $+\mathrm{HBO}_{2 \mathrm{~W}}, \mathrm{SCI}+\mathrm{HBO}_{4 \mathrm{~W}}$, and $\mathrm{SCI}+\mathrm{HBO}_{6 \mathrm{~W}}$ groups, were significantly higher than that of the SCI group but lower than the SH group (all $P<0.001$ ). The BBB score of the $\mathrm{SCI}+\mathrm{HBO}_{4 \mathrm{~W}}$ group was significantly higher than that of the $\mathrm{SCI}+\mathrm{HBO}_{2 \mathrm{~W}}$ group $(P<0.001)$, but there was no significant difference of $\mathrm{BBB}$ scores between the $\mathrm{SCI}+\mathrm{HBO}_{4 \mathrm{~W}}$ and $\mathrm{SCI}+\mathrm{HBO}_{6 \mathrm{~W}}$ groups (Fig. 1).

\section{Conventional MRI findings}

As shown in Fig. 2, cavities were formed in the lesion site of the injured spinal cord which were/weren't accompanied with syringomyelia in rats from the SCI and SCI+HBO groups, showing low signal intensity on T1-weighted images and high signal intensity on T2-weighted images.

\section{DTI parameters}

FA, MD, RD, and AD values of rats in each group are shown in Table 1 and Fig. 3. Lower FA but higher MD and RD values at the epicenter, rostral and caudal levels were found in the SCI group than those in the SH group (all $P<0.001$ ). Higher FA values at the epicenter, rostral and caudal levels were found in the $\mathrm{SCI}+\mathrm{HBO}_{4 \mathrm{~W}}$ group than those in the $\mathrm{SCI}$ and $\mathrm{SCI}+\mathrm{HBO}_{2 \mathrm{~W}}$ groups (all $P<$ $0.05)$, whereas no significant differences were observed between the $\mathrm{SCI}+\mathrm{HBO}_{4 \mathrm{~W}}$ and $\mathrm{SCI}+\mathrm{HBO}_{6 \mathrm{~W}}$ groups. MD 
and RD values at the epicenter, rostral, and caudal levels of the $\mathrm{SCI}+\mathrm{HBO}_{4 \mathrm{~W}}$ group were significantly lower than the SCI group (all $P<0.01$ ). Meanwhile, there were no significant differences between any groups for $\mathrm{AD}$ values.

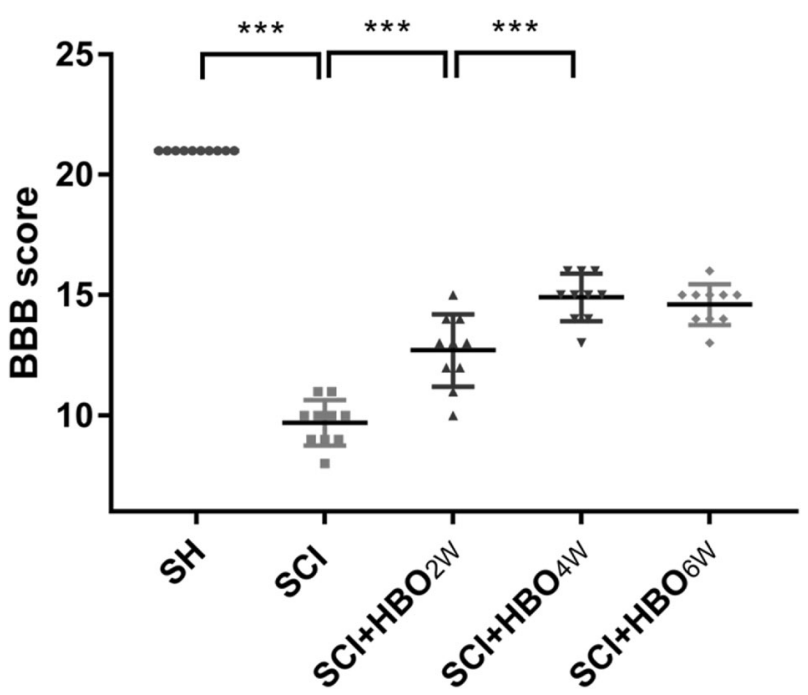

Fig. 1 Basso Beattie Bresnahan (BBB) scores for hind limb motor function in rats from the SH, SCI, and different time course of SCI $+\mathrm{HBO}$ groups. SH, sham-operated; SCI, spinal cord injury; SCI $+\mathrm{HBO}_{2 \mathrm{~W}}$, spinal cord injury and hyperbaric oxygen therapy for 2 weeks group; $\mathrm{SCI}+\mathrm{HBO}_{4 \mathrm{~W}}$, spinal cord injury and hyperbaric oxygen therapy for 4 weeks group; $\mathrm{SCI}+\mathrm{HBO}_{6 \mathrm{~W}}$, spinal cord injury and hyperbaric oxygen therapy for 6 weeks group. ${ }^{* * *} P<0.001$

\section{Correlation between DTI parameters and BBB scores}

Pearson correlation analysis indicated that FA values showed positive correlations with the BBB scores $(r=$ 0.941, 0.948, and 0.946 for epicenter, rostral, and caudal levels, respectively; all $P<0.001$ ), while $\mathrm{MD}$ and $\mathrm{RD}$ values were negatively correlated with the BBB scores (epicenter: $r=-0.841$ and -0.800 ; rostral: $r=-0.759$ and -0.800 ; caudal: $r=-0.754$ and -0.793 ; all $P<0.001$ ). However, $\mathrm{AD}$ values had no correlations with the $\mathrm{BBB}$ scores (Fig. 4).

\section{Discussion}

In our study, the lower limb function of rats in the SCI $+\mathrm{HBO}$ groups (including $\mathrm{SCI}+\mathrm{HBO}_{2 \mathrm{~W}}, \mathrm{SCI}+\mathrm{HBO}_{4 \mathrm{~W}}$, and $\mathrm{SCI}+\mathrm{HBO}_{6 \mathrm{~W}}$ groups) indicated by $\mathrm{BBB}$ score had achieved significant improvement than that of SCI group, showing that $\mathrm{HBO}$ treatment following $\mathrm{SCI}$ in rats exerts a protective effect for the recovery of locomotor function, which was consistent with previous studies [17]. Besides, Li et al [18]. found that months after SCI, the spinal cord caudal to the injury epicenter was in a chronic state of hypoxia resulting from local vessel constriction induced by pericytes, which was one of the most significant mechanisms involved in secondary injury after SCI. Hence, an increase in inhaled oxygen can produce substantial relief from hypoxia and improve motoneuron and locomotor function after SCI.
Fig. 2 Cavities were formed in the lesion site of injured spinal cord of rats from the SCI and $\mathrm{SCI}+\mathrm{HBO}$ groups showing high signal intensity on T2WI and low signal intensity on T1WI accompanied with syringomyelia (A1-A2) or not (B1-D2). SCI, spinal cord injury; $\mathrm{SCI}+\mathrm{HBO}_{2 \mathrm{~W}}$, spinal cord injury and hyperbaric oxygen therapy for 2 weeks group; $\mathrm{SCI}+\mathrm{HBO}_{4 \mathrm{~W}}$, spinal cord injury and hyperbaric oxygen therapy for 4 weeks group; $\mathrm{SCI}+\mathrm{HBO}_{6 \mathrm{~W}}$, spinal cord injury and hyperbaric oxygen therapy for 6 weeks group
$\mathrm{SCl}$
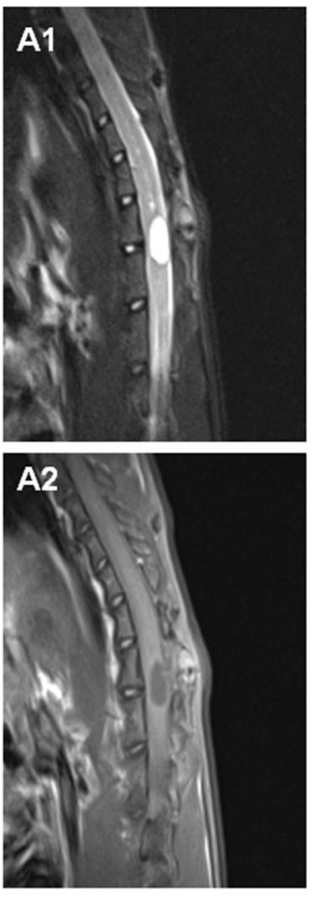

$\mathrm{SCl}+\mathrm{HBO}_{2} \mathrm{~W}$
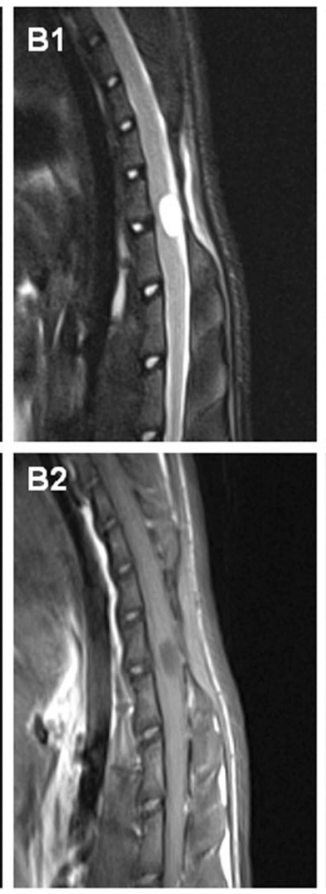

$\mathrm{SCl}+\mathrm{HBO}_{4}$
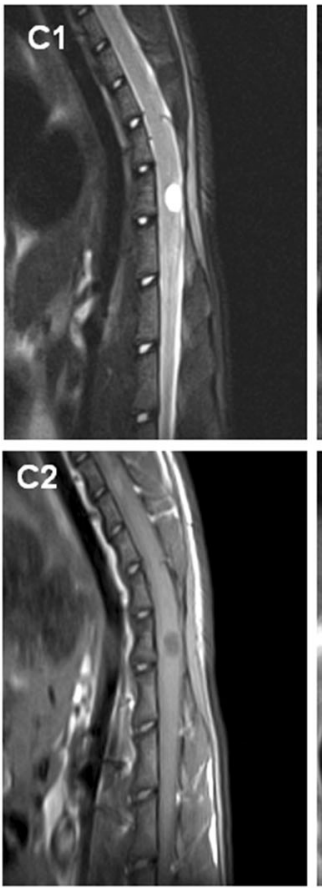

$\mathrm{SCl}+\mathrm{HBO}_{6 \mathrm{~W}}$
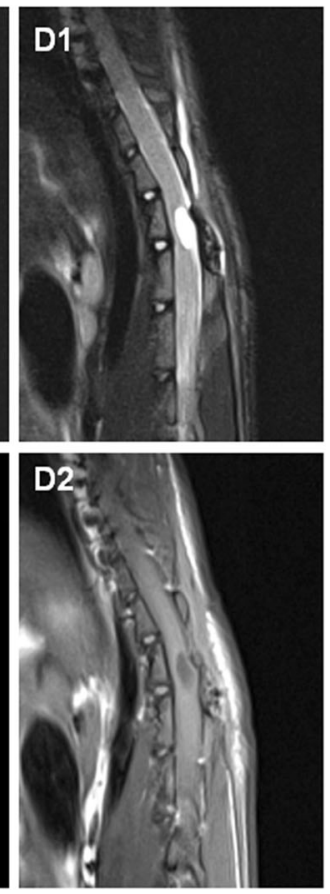
Table $1 \mathrm{FA}, \mathrm{MD}, \mathrm{RD}$, and $\mathrm{AD}$ values of rats in $\mathrm{SH}, \mathrm{SCI}$, and different time course of $\mathrm{SCI}+\mathrm{HBO}$ groups

\begin{tabular}{|c|c|c|c|c|}
\hline & FA & $\begin{array}{l}\mathrm{MD}\left(\times 10^{-3}\right. \\
\left.\mathrm{mm}^{2} / \mathrm{s}\right)\end{array}$ & $\begin{array}{l}\mathrm{RD}\left(\times 10^{-3}\right. \\
\left.\mathrm{mm}^{2} / \mathrm{s}\right)\end{array}$ & $\begin{array}{l}\mathrm{AD}\left(\times 10^{-3}\right. \\
\left.\mathrm{mm}^{2} / \mathrm{s}\right)\end{array}$ \\
\hline \multicolumn{5}{|l|}{ SH } \\
\hline Epicenter & $0.56 \pm 0.01$ & $0.92 \pm 0.09$ & $0.80 \pm 0.11$ & $2.15 \pm 0.12$ \\
\hline Rostral & $0.56 \pm 0.01$ & $0.95 \pm 0.08$ & $0.81 \pm 0.10$ & $2.17 \pm 0.16$ \\
\hline Caudal & $0.56 \pm 0.01$ & $0.95 \pm 0.08$ & $0.80 \pm 0.11$ & $2.19 \pm 0.10$ \\
\hline \multicolumn{5}{|l|}{$S C I$} \\
\hline Epicenter & $0.33 \pm 0.03$ & $1.48 \pm 0.16$ & $1.16 \pm 0.08$ & $1.96 \pm 0.27$ \\
\hline Rostral & $0.36 \pm 0.03$ & $1.33 \pm 0.09$ & $1.14 \pm 0.08$ & $2.03 \pm 0.25$ \\
\hline Caudal & $0.36 \pm 0.03$ & $1.33 \pm 0.16$ & $1.13 \pm 0.09$ & $2.01 \pm 0.23$ \\
\hline \multicolumn{5}{|c|}{$S C I+H B O_{2 W}$} \\
\hline Epicenter & $0.41 \pm 0.05$ & $1.28 \pm 0.18$ & $0.98 \pm 0.18$ & $1.81 \pm 0.30$ \\
\hline Rostral & $0.43 \pm 0.05$ & $1.16 \pm 0.15$ & $0.97 \pm 0.16$ & $1.83 \pm 0.28$ \\
\hline Caudal & $0.43 \pm 0.04$ & $1.17 \pm 0.18$ & $0.98 \pm 0.15$ & $1.86 \pm 0.25$ \\
\hline \multicolumn{5}{|c|}{$S C I+H_{B O} O_{4 W}$} \\
\hline Epicenter & $0.47 \pm 0.04$ & $1.09 \pm 014$ & $0.86 \pm 0.16$ & $1.79 \pm 0.32$ \\
\hline Rostral & $0.50 \pm 0.04$ & $0.99 \pm 0.13$ & $0.87 \pm 0.15$ & $1.86 \pm 0.31$ \\
\hline Caudal & $0.50 \pm 0.04$ & $0.96 \pm 0.11$ & $0.86 \pm 0.16$ & $1.86 \pm 0.31$ \\
\hline \multicolumn{5}{|c|}{$S C I+H B O_{6 W}$} \\
\hline Epicenter & $0.47 \pm 0.03$ & $1.08 \pm 0.13$ & $0.85 \pm 0.08$ & $1.78 \pm 0.30$ \\
\hline Rostral & $0.50 \pm 0.02$ & $0.98 \pm 0.13$ & $0.88 \pm 0.09$ & $1.84 \pm 0.30$ \\
\hline Caudal & $0.49 \pm 0.02$ & $1.00 \pm 0.11$ & $0.87 \pm 0.09$ & $1.86 \pm 0.20$ \\
\hline
\end{tabular}

$S H$ sham-operated, $S C I$ spinal cord injury, $S C I+H B O_{2 W}$ spinal cord injury and hyperbaric oxygen therapy for 2 weeks group, $S C I+H B O_{4 W}$ spinal cord injury and hyperbaric oxygen therapy for 4 weeks group, $S C I+H B O_{6 W}$ spinal cord injury and hyperbaric oxygen therapy for 6 weeks group, $F A$ fractional anisotropy, $M D$ mean diffusivity, $R D$ radial diffusion, $A D$ axial diffusion

Furthermore, experimental studies investigating the effect of $\mathrm{HBO}$ therapy proposed that the neuroprotection provided by HBO therapy involves the following several mechanisms: (1) decreasing apoptosis; (2) diminishing inflammation; (3) reducing oxidative stress; (4) promoting angiogenesis; (5) reducing spinal cord edema; (6) increasing autophagy [19].

In the present study, motor function of rats in the SCI $+\mathrm{HBO}_{4 \mathrm{~W}}$ group had significant improvement compared to that in the $\mathrm{SCI}$ and $\mathrm{SCI}+\mathrm{HBO}_{2 \mathrm{~W}}$ groups, whereas no significant neurologic recovery for rats between the SCI $+\mathrm{HBO}_{4 \mathrm{~W}}$ and the $\mathrm{SCI}+\mathrm{HBO}_{6 \mathrm{~W}}$ groups were observed, indicating that the prolonged course after 4 weeks of HBO treatment contribute little to improve the motor recovery of rats after SCI. Therefore, we proposed that 4 weeks may be an more appropriate course of HBO therapy for rats with SCI. Apoptosis (programmed cell death) is a major cause of post-injury neuronal death and plays a pivotal role in the secondary damage $[20,21]$. In a study reported by Crowe et al. [22], apoptotic cells were found from $6 \mathrm{~h}$ to 3 weeks after injury. Therefore, the prevention of neuronal death by
HBO therapy should be activated mainly from $6 \mathrm{~h}$ to 3 weeks after SCI in regard to the mechanism of apoptosis. As to inflammation, another hallmark of secondary spinal injury, the result of study conducted by Beck et al [23]. demonstrated a novel time-dependent multiphasic response of cellular inflammation in the spinal cord after SCI. The early phase of cellular inflammation is mostly comprised of neutrophils (peaking 1 day post-injury), macrophages/ microglia (peaking 7 days post-injury) and $\mathrm{T}$ cells (peaking 9 days post-injury). The late phase of cellular inflammation was detected after 14 days post-injury, peaked after 60 days post-injury and remained detectable throughout 180 days post-injury for all three cell types. However, the late phase of cellular inflammation (14-180 days post-injury) had little effect on locomotor function after SCI. In a word, the inflammation that responsible for aggravating injury mainly occurred in the first 14 days. Moreover, Yiu et al. [24] found that glial scar was a major hurdle for successful axon regeneration, weakening the effect of HBO treatment. Kjell et al. [25] reported that reactive astrocytes cluster at the border of the lesion 1-2 weeks after injury and the glial scar has matured after 2-3 weeks in rats. Thus, HBO therapy within the critical point, 3 weeks after injury, is greatly important for alleviating secondary injury and improving neurological functional. In other words, the prolonged course after 3 weeks of HBO treatment may have limited effect in improving the motor recovery of individuals after SCI, which can explain why 4 weeks of HBO therapy was a more appreciate choice than 2 weeks and 6 weeks in our study.

Our study demonstrated that FA values at the epicenter, rostral and caudal levels significantly decreased after SCI. In injured spinal cords, the destruction, necrosis of axonal nerve fibers and demyelination resulting from primary and secondary damage contribute to the decrease of the anisotropy and eventually lead to the decreased FA values [26, 27]. In our study, we also observed the differences of MD and RD values between the SCI and SH groups. In the study of Zhao et al. [28], MD values of rats increased 84 days after SCI, which thought to be related to the large amount of cerebrospinal fluid in the cavity strengthening the diffusion degree of water molecules, and demyelination spread from the epicenter to distal ends along with secondary injury during the chronic stage, resulting in an increased RD value consequently. The results of this study showed that FA values were higher in the $\mathrm{SCI}+\mathrm{HBO}$ groups and MD and $\mathrm{RD}$ values were lower in the $\mathrm{SCI}+\mathrm{HBO}_{4 \mathrm{w}}$ group than those in the SCI group. Seo et al. [29] found that and neuronal and glial apoptosis was a key feature of SCI. HBO therapy can reduce hypoxia-induced neuronal and glial apoptosis, which was related to axonal degeneration and demyelination [22], by elevating the blood oxygen concentration in the injured spinal cord. Therefore, HBO therapy can reduce the 


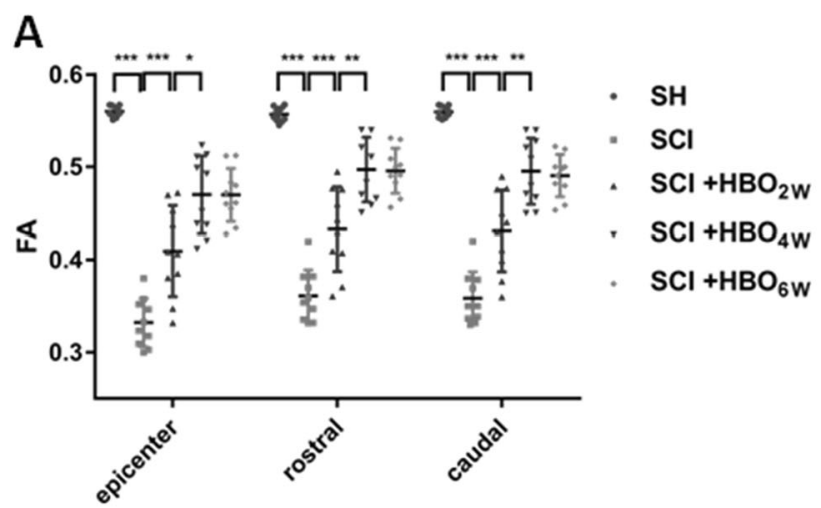

C

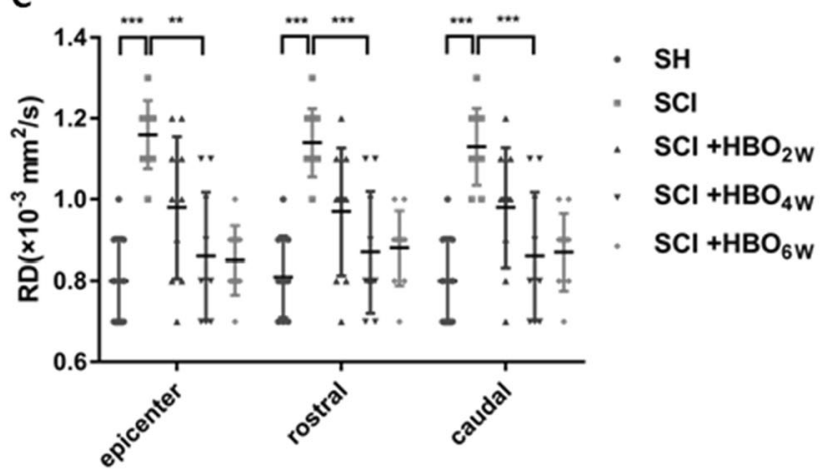

Fig. 3 FA (a), MD (b), RD (c), and AD (d) values at the epicenter, rostral and caudal levels of rats in SH, SCI and different time course of $\mathrm{SCI}+\mathrm{HBO}$ groups. $\mathrm{SH}$, sham-operated; SCI, spinal cord injury; SCI $+\mathrm{HBO}_{2 \mathrm{~W}}$, spinal cord injury and hyperbaric oxygen therapy for 2 weeks group; $\mathrm{SCI}+\mathrm{HBO}_{4 \mathrm{~W}}$, spinal cord injury and hyperbaric

destruction for spared spinal tissue surviving from the initial trauma. Furthermore, the study conducted by Huang et al. [30] also confirmed that the injured spinal cord, treated with $\mathrm{HBO}$ in the early post-injury stage, possessed more neurons in gray matter and more sparing myelin tissue in white matter than untreated cords, which can be shown with a higher FA values and lower MD and RD values after $\mathrm{HBO}$ therapy. A previous study showed that the residual white matter tract had a significant effect on the spontaneous locomotor recovery [31]. In this study we found that DTI parameters, especially FA values, exhibited a strong correlation with BBB scores. Additionally, FA values at the epicenter, rostral and caudal levels had a good consistency with BBB scores in comparing the efficacy of $\mathrm{HBO}$ therapy in rat after SCI with different treatment course, indicating that DTI parameters may serve as efficient biomarkers to reflect the functional recovery after HBO therapy and to seek for the better course for HBO therapy in rats with SCI.

There are some limitations in this study. First, the histopathological analysis was not performed. Second, all MRI examinations were performed on a 3.0T MRI scanner,

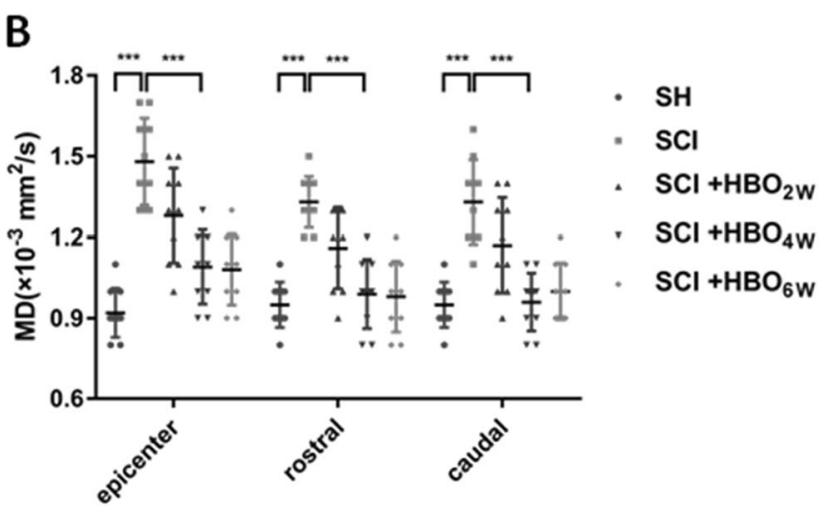

D

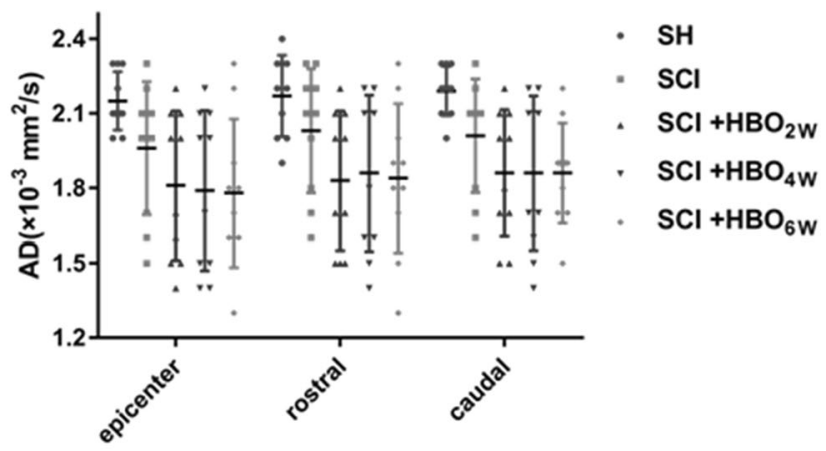

oxygen therapy for 4 weeks group; $\mathrm{SCI}+\mathrm{HBO}_{6 \mathrm{~W}}$, spinal cord injury and hyperbaric oxygen therapy for 6 weeks group. FA, fractional anisotropy; $\mathrm{MD}$, mean diffusivity; $\mathrm{RD}$, radial diffusion; $\mathrm{AD}$, axial diffusion. ${ }^{* * *} P<0.001,{ }^{* *} P<0.01, * P<0.05$

therefore, the DTI parameters could not be collected from the white matter and gray matter respectively due to the quality of acquired images and the relatively small size of the rodent spinal cord. Third, although there were potential differences in BBB scores of rats immediately after SCI, the randomization of grouping was performed in our initial study in order to reduce the differences between groups. Fourth, the sham-operated group was added in the later stage on the request from a reviewer, thus, rats in this group were not randomized and were tested at a different time. Finally, we deduced the more appropriate course for HBO therapy after SCI based on animal experiments, further studies in human beings are required to validate our finding.

In conclusion, HBO treatment is beneficial for neurological recovery when applied in rats after SCI and 4 weeks may be the more appropriate course for HBO therapy. DTI parameters, especially FA values, could noninvasively and quantifiably evaluate the efficacy of HBO treatment for rats with SCI. Further study will be required to elucidate whether the better course for HBO therapy in rats after SCI is affected by the degree of injury severity and the HBO protocols. 
A

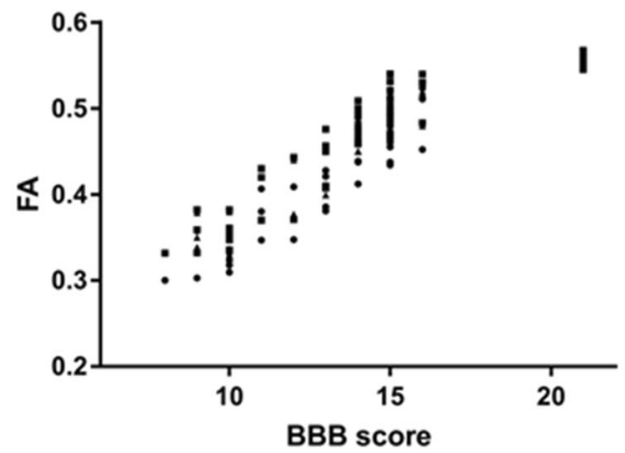

C

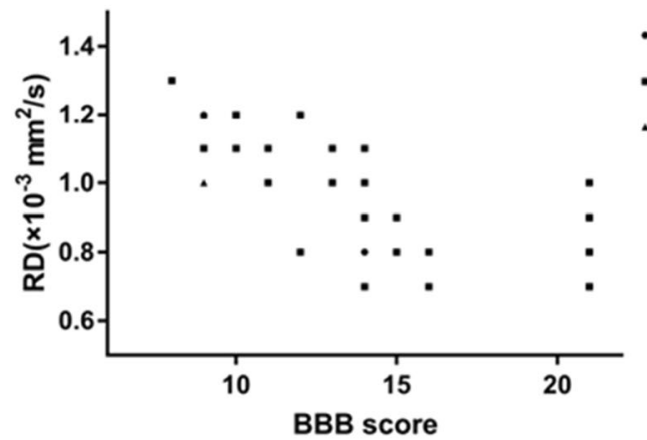

B

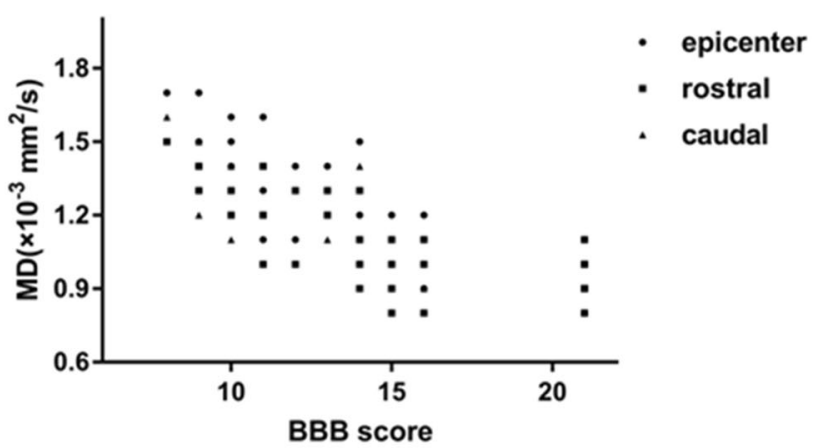

D

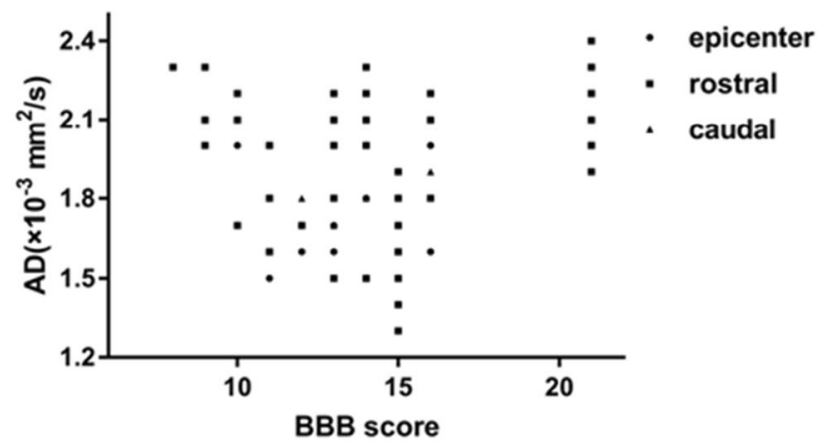

Fig. 4 Variation trend of FA (a), MD (b), RD (c), and $\mathrm{AD}(\mathbf{d})$ values at the epicenter, rostral and caudal levels and Basso Beattie Bresnahan (BBB) score. FA, fractional anisotropy; $\mathrm{MD}$, mean diffusivity; $\mathrm{RD}$, radial diffusion; $\mathrm{AD}$, axial diffusion

\section{Data archiving}

The datasets generated and/or analysed during the current study are available from the corresponding author on reasonable request.

Acknowledgements This work was supported by the Natural Science Foundation of Fujian Province (No. 2016J01526).

Funding This work was supported by the Natural Science Foundation of Fujian Province (No. 2016J01526).

Author contributions Fang Liu: Project development and assistance with manuscript writing; Libin Yang: Assistance with experimental operation, data collection and manuscript writing; Jianyi Liu: Assistance with experimental operation; Yijing Zhao: Assistance with experimental operation; Zebin Xiao: Assistance with manuscript writing; Yingyan Zheng: Assistance with manuscript preparation; Zhen Xing: Assistance with manuscript preparation; Yuyang Zhang: Assistance with manuscript preparation; Dairong Cao: Project development and assistance with manuscript writing.

\section{Compliance with ethical standards}

Ethical approval We certify that all applicable institutional and governmental regulations concerning the ethical use of animals were followed during the course of this research.
Conflict of interest The authors declare that they have no conflict of interest.

Publisher's note: Springer Nature remains neutral with regard to jurisdictional claims in published maps and institutional affiliations.

\section{References}

1. Jazayeri SB, Beygi S, Shokraneh F, Hagen EM, RahimiMovaghar V. Incidence of traumatic spinal cord injury worldwide: a systematic review. Eur Spine J. 2015;24:905-18.

2. Kwon BK, Tetzlaff W, Grauer JN, Beiner J, Vaccaro AR. Pathophysiology and pharmacologic treatment of acute spinal cord injury. Spine J. 2004;4:451-64.

3. Long Y, Liang F, Gao CJ, Li Z, Yang J. Hyperbaric oxygen therapy reduces apoptosis after spinal cord injury in rats. Int J Clin Exp Med. 2014;7:4073-81.

4. Tan J, Zhang F, Liang F, Wang Y, Li Z, Yang J, et al. Protective effects of hyperbaric oxygen treatment against spinal cord injury in rats via toll-like receptor $2 /$ nuclear factor- $\kappa B$ signaling. Int $J$ Clin Exp Pathol. 2014;7:1911-9.

5. Wang Y, Zhang S, Luo M, Li Y. Hyperbaric oxygen therapy improves local microenvironment after spinal cord injury. Neural Regen Res. 2014;9:2182-8.

6. Kang N, Hai Y, Yang J, Liang F, Gao CJ. Hyperbaric oxygen intervention reduces secondary spinal cord injury in rats via regulation of HMGB1/TLR4/NF- $\mathrm{KB}$ signaling pathway. Int $\mathrm{J}$ Clin Exp Pathol. 2015;8:1141-53. 
7. Sun Y, Liu D, Su P, Lin F, Tang Q. Changes in autophagy in rats after spinal cord injury and the effect of hyperbaric oxygen on autophagy. Neurosci Lett. 2016;618:139-45.

8. Mhuircheartaigh NN, Kerr JM, Murray JG. MR imaging of traumatic spinal injuries. Semin Musculoskelet Radiol. 2006;10:293-307.

9. Loy DN, Kim JH, Xie M, Schmidt RE, Trinkaus K, Song S-K. Diffusion tensor imaging predicts hyperacute spinal cord injury severity. J Neurotrauma. 2007;24:979-90.

10. Kelley BJ, Harel NY, Kim CY, Papademetris X, Coman D, Wang $\mathrm{X}$, et al. Diffusion tensor imaging as a predictor of locomotor function after experimental spinal cord injury and recovery. $\mathrm{J}$ Neurotrauma. 2014;31:1362-73.

11. Li XH, Li JB, He XJ, Wang F, Huang SL, Bai ZL. Timing of diffusion tensor imaging in the acute spinal cord injury of rats. Sci Rep. 2015;5:12639.

12. Patel SP, Smith TD, VanRooyen JL, Powell D, Cox DH, Sullivan $\mathrm{PG}$, et al. Serial diffusion tensor imaging in vivo predicts longterm functional recovery and histopathology in rats following different severities of spinal cord injury. J Neurotrauma. 2016;33:917-28.

13. Sun W, Tan J, Li Z, Lu S, Li M, Kong C, et al. Evaluation of hyperbaric oxygen treatment in acute traumatic spinal cord injury in rats using diffusion tensor imaging. Aging Dis. 2018;9:391-400.

14. Budde MD, Xie M, Cross AH, Song SK. Axial diffusivity is the primary correlate of axonal injury in the experimental autoimmune encephalomyelitis spinal cord: a quantitative pixelwise analysis. J Neurosci. 2009;29:2805-13.

15. Janve VA, Zu Z, Yao SY, Li K, Zhang FL, Wilson KJ, et al. The radial diffusivity and magnetization transfer pool size ratio are sensitive markers for demyelination in a rat model of type III multiple sclerosis (MS) lesions. Neuroimage. 2013;74:298-305.

16. Basso DM, Beattie MS, Bresnahan JC. Graded histological and locomotor outcomes after spinal cord contusion using the NYU weight-drop device versus transection. Exp Neurol. 1996; 139:244-56.

17. Falavigna A, Figueiro MP, Silva PGD, Conzatti LP, Rizkalla EB, Santos SCD, et al. Hyperbaric oxygen therapy after acute thoracic spinal cord injury: improvement of locomotor recovery in rats. Spine. 2018;43:E442-E7.

18. Li Y, Lucas-Osma AM, Black S, Bandet MV, Stephens MJ, Vavrek R, et al. Pericytes impair capillary blood flow and motor function after chronic spinal cord injury. Nat Med. 2017;23: 733-41.

19. Patel NP, Huang JH. Hyperbaric oxygen therapy of spinal cord injury. Med Gas Res. 2017;7:133-43.

20. Beattie MS, Farooqui AA, Bresnahan JC. Review of current evidence for apoptosis after spinal cord injury. J Neurotrauma. 2000;17:915-25.

21. Lu J, Ashwell KW, Waite P. Advances in secondary spinal cord injury: role of apoptosis. Spine. 2000;25:1859-66.

22. Crowe MJ, Bresnahan JC, Shuman SL, Masters JN, Beattie MS. Apoptosis and delayed degeneration after spinal cord injury in rats and monkeys. Nat Med. 1997;3:73-6.

23. Beck KD, Nguyen HX, Galvan MD, Salazar DL, Woodruff TM, Anderson AJ. Quantitative analysis of cellular inflammation after traumatic spinal cord injury: evidence for a multiphasic inflammatory response in the acute to chronic environment. Brain. 2010;133:433-47.

24. Yiu G, He Z. Glial inhibition of CNS axon regeneration. Nat Rev Neurosci. 2006;7:617-27.

25. Kjell J, Olson L. Rat models of spinal cord injury: from pathology to potential therapies. Dis Model Mech. 2016;9:1125-37.

26. Song S-K, Sun S-W, Ramsbottom MJ, Chang C, Russell J, Cross AH. Dysmyelination revealed through MRI as increased radial (but unchanged axial) diffusion of water. Neuroimage. 2002;17:1429-36.

27. Mori S, Zhang J. Principles of diffusion tensor imaging and its applications to basic neuroscience research. Neuron. 2006;51:527-39.

28. Zhao C, Rao JS, Pei XJ, Lei JF, Wang ZJ, Zhao W, et al. Diffusion tensor imaging of spinal cord parenchyma lesion in rat with chronic spinal cord injury. Magn Reson Imaging. 2018;47:25-32.

29. Seo JY, Kim YH, Kim JW, Kim SI, Ha KY. Effects of therapeutic hypothermia on apoptosis and autophagy after spinal cord injury in rats. Spine. 2015;40:883-90.

30. Huang L, Mehta M, Nanda A, Zhang J. The role of multiple hyperbaric oxygenation in expanding therapeutic windows after acute spinal cord injury in rats. J Neurosurg. 2003; 99:198-205.

31. Kim JH, Song SK, Burke DA, Magnuson DS. Comprehensive locomotor outcomes correlate to hyperacute diffusion tensor measures after spinal cord injury in the adult rat. Exp Neurol. 2012;235:188-96. 